\title{
Evidence of the coupling of a fast magnetospheric cavity mode to field line resonances
}

\author{
Ching-Chang Cheng ${ }^{1,2}$, Jih-Kwin $\mathrm{Chao}^{2}$, and Tung-Shin $\mathrm{Hsu}^{3}$ \\ ${ }^{1}$ Department of Physics, National Hu-Wei Institute of Technology, Hu-Wei, Taiwan, R.O.C. \\ ${ }^{2}$ Institute of Space Science, National Central University, Chung-Li, Taiwan, R.O.C. \\ ${ }^{3}$ IGPP, University of California in Los Angeles, Los Angeles, U.S.A.
}

(Received March 17, 1997; Revised February 28, 1998; Accepted May 1, 1998)

\begin{abstract}
We present an evidence of the coupling of a fast magnetospheric cavity mode to field line resonances. The power of filtered $H$ and $D$ components of Pi 2 pulsations during March-April in 1978 and 1979 from IGS chain in the United Kingdom (UK) shows that there are two enhancements located near $L=3.5$ and 5.5 in the distribution profile. The irreversible hydromagnetic wave coupling is studied by using a box model for the magnetosphere. The cold linearized MHD equations is examined with realistic Alfven speed profile for the uniform and nonuniform ambient magnetic fields, respectively. With appropriate parameters to depict the magnetospheric environments during the aforementioned period, numerical results are acquired with the fourth order Runge-Kutta method and quite consistent with data analysis. We suggest that the coupling of a fast magnetospheric cavity mode driven by fast compressional waves owing to the impulsive source at the magnetotail to resonant field lines at low latitudes is a possible scenario of the propagation mechanism for low latitude Pi 2 pulsations.
\end{abstract}

\section{Introduction}

The sources of magnetohydrodynamic (MHD) waves in the magnetosphere are generally believed to be the plasma instabilities or external disturbances by the impinging solar wind (see review by Allan and Poulter, 1992). MHD waves have been studied by using ground-based and space borne instrumentations. They are called geomagnetic pulsations and classified as Pc (continuous pulsations) and Pi (irregular pulsations) due to their morphological characteristics (e.g. Jacobs et al., 1964). How they propagate in the inner magnetosphere and how their frequencies are selected still remain to be investigated.

There are two decoupled modes of MHD waves in the homogeneous cold plasma. One is called shear Alfven waves (or the toroidal mode) which can propagate along the ambient magnetic field. The other is fast compressional waves (or the poloidal mode) which can traverse the ambient magnetic field. In the magnetosphere, shear Alfven waves travel along the ambient magnetic field and reflect back from the highlyconducting ionosphere. As a result, the standing oscillation of the ambient field line could be driven. On the other hand, fast compressional waves may bounce back from the low latitude ionosphere which could be a perfect conductor. The cavity modes resonance would occur as fast compressional waves are trapped in the magnetospheric cavity. With analogue of the field line to a string, a standing oscillation in the field line may have the harmonic frequency. In addition, cavity modes are characterized by the discrete spectrum as dependent on the size of the magnetospheric cavity. Due to the inhomogeneous distribution of the plasma and field con-

Copy right(C) The Society of Geomagnetism and Earth, Planetary and Space Sciences (SGEPSS); The Seismological Society of Japan; The Volcanological Society of Japan; The Geodetic Society of Japan; The Japanese Society for Planetary Sciences. figurations in the magnetosphere, fast compressional waves may couple to shear Alfven waves and cavity resonances would excite the resonant field line as the wave frequency is matched. In other words, the coupling of fast magnetospheric cavity modes to field line resonances may occur as the eigenfrequency meets.

To explain latitude-dependent magnetic pulsations at the higher latitudes, field line resonances (FLRs) is widely believed to be a crucial mechanism in the wave coupling as evanescent surface compressional waves generated owing to the Kelvin-Helmholtz instabilities at the dayside magnetopause tunnel to excite the field line oscillations in the inner magnetosphere (Chen and Hasegawa, 1974; Southwood, 1974). In addition, early observations (e.g. Samson and Rostoker, 1972) reported that magnetic pulsations had the characteristics of discrete spectrum and suggested that there might exist global cavity modes. Recent observations pointed out that global cavity modes may be responsible for magnetic pulsations at very low latitudes and in the inner magnetosphere (Lin et al., 1991; Takahashi et al., 1995). However, some studies suggested that the coupling of global cavity modes to FLR is likely responsible for magnetic pulsations at very low latitudes (Feng et al., 1995). Moreover, many theoretical studies and numerical works have been focused on the spatial structure of the coupling of global cavity modes to FLRs (e.g. Allan et al., 1986; Kivelson and Southwood, 1986; Krauss-Varban and Patel, 1988; Zhu and Kivelson, 1989; Fujita and Patel, 1992; Lee, 1996; and references there in). It is noteworthy that there are few observational evidences to show the coupling of fast magnetospheric cavity modes to FLRs except in theoretical and numerical studies.

The main goal of this study is to present an observational 


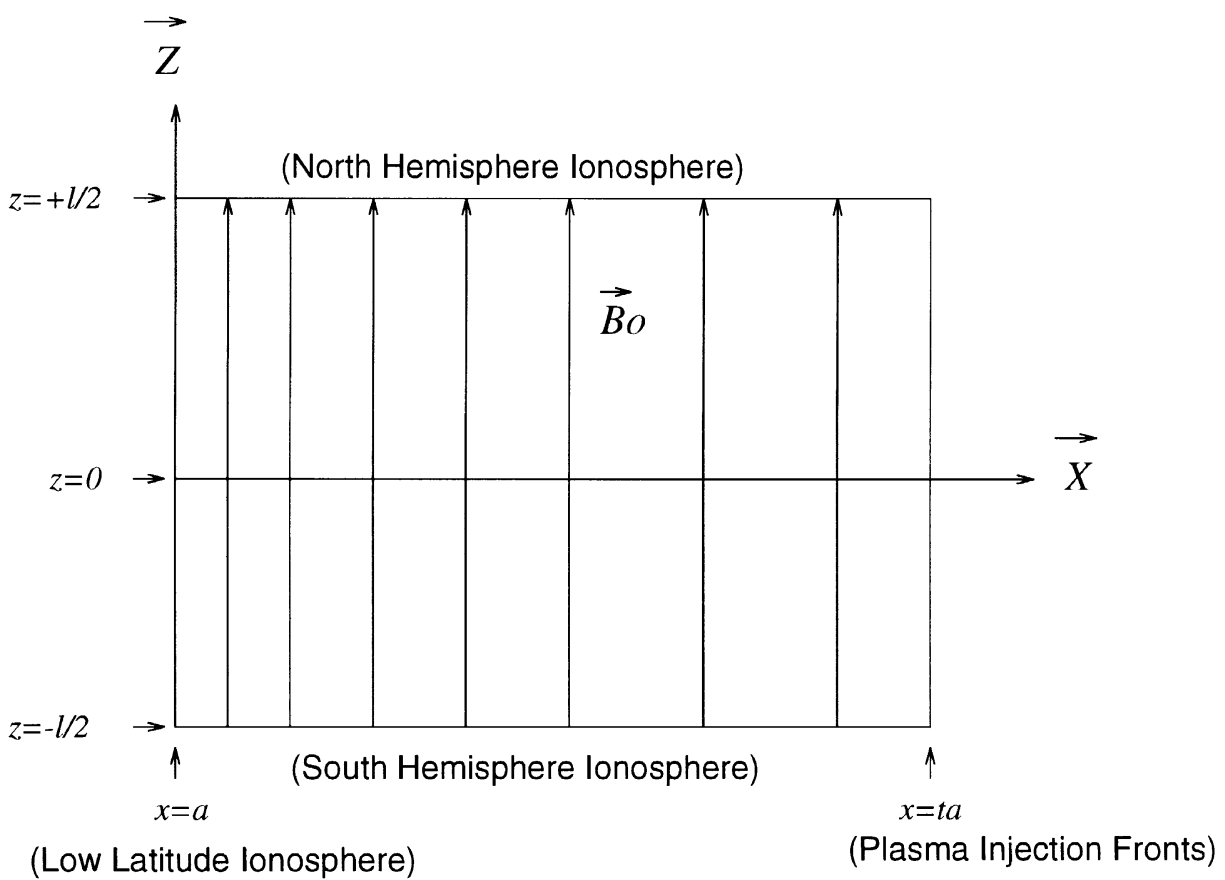

Fig. 1. The box geometry used to illustrate the nightside magnetospheric cavity.

evidence of the coupling of a fast magnetospheric cavity mode to FLRs. Recently, Hsu and McPherron (1996) have been trying to develop a database to clarify the substorm mechanism. They visually inspected the AL index to choose every possible substorm-like event. Based on their selection criterion (Hsu and McPherron, 1996), almost a thousand events have been identified visually. In order to get more precise substorm onset timing, they use Pi 2 pulsations as a second-order improvement for AL onset timing. Pi 2 pulsations are impulsive, damped oscillations of geomagnetic field in the period range $40-150$ seconds. This type of pulsations is known to be associated with substorm onset (Saito et al., 1976). In addition, Pi 2 pulsations are predominantly a nighttime phenomena (Yeoman et al., 1994). Hsu and McPherron (1996) went through Pi 2 pulsations of March-April in 1978 and 1979 from IGS (cf. Stuart, 1982) chain because of the available ISEE 2 plasma data. In their study, the $\mathrm{Pi} 2$ power is obtained with the combination of the power of filtered $H$ and $D$ components. The Pi 2 power in IGS chain shows that there are two enhancements located near $L=3.5$ and 5.5, respectively. It is generally known that $H$ and $D$ components of geomagnetic pulsations are correlated to the compressional and toroidal components of MHD waves in the magnetosphere. There are three possible source models for Pi 2 pulsations at lower latitudes (Yeoman and Orr, 1989): low latitude FLRs driven by fast compressional waves originating from auroral oscillating field lines or the substorm current wedge, surface waves at the plasmapause, a magnetospheric/plasmaspheric cavity resonances. None of these source models can explain the power distribution of Pi 2 pulsations in IGS chain. At the substorm expansive onset, hydromagnetic disturbances driven by plasma injection may propagate across the ambient magnetic field and excite cavity-resonance in the inner magnetosphere. Hence, Yumoto et al. (1989) proposed that the magnetosphere cavity resonance may further couple to resonant oscillation of field lines at lower latitudes. To clarify whether the observational result can be accounted for by the coupling of a fast magnetospheric cavity mode to FLRs, we have to examine the cold linearized MHD equations with the realistic Alfven speed profile in the theoretical model.

\section{The Theoretical Model}

As a preliminary step to investigate the coupling of a fast magnetospheric cavity mode to FLRs, the magnetospheric cavity is simplified as a box. Unlike the cylinder and dipole models, the box model is mathematically tractable to quantitatively compare with the observational result. The Cartesian coordinates are used to describe the box model. Let the origin point locate at the center of the Earth and the $+x$ axis directed antisunward. The direction of the $+z$ axis is along the Earth's magnetic fields. Finally, the $+y$ axis is directed toward the dawn. The radial distance $x$ is in unit of the Earth's radius $a$ such that $x=a$ is the ionosphere at the low latitude and $x=t a$ corresponds to plasma injection fronts initiated by the tail reconnection at the substorm expansive onset (see Fig. 1). Although the modeling of the magnetospheric substorm is still a controversial issue (Fairfield, 1992), the classical near-earth neutral line model is a well popular one. At the substorm expansive onset, the X-line is formed at $x \sim 10 a$ in the tail (e.g. McPherron et al., 1973). The tail reconnection would result from the formation of a X-line and launch plasma injection. Hence, $x=t a=10 a$ corresponding to the site of plasma injection fronts can be assumed as the tail boundary in this study. In the box model, $l$ denotes the length of the ambient magnetic field line. In Fig. $1, z=l / 2$ corresponds to the north hemisphere ionosphere and $z=-l / 2$ 


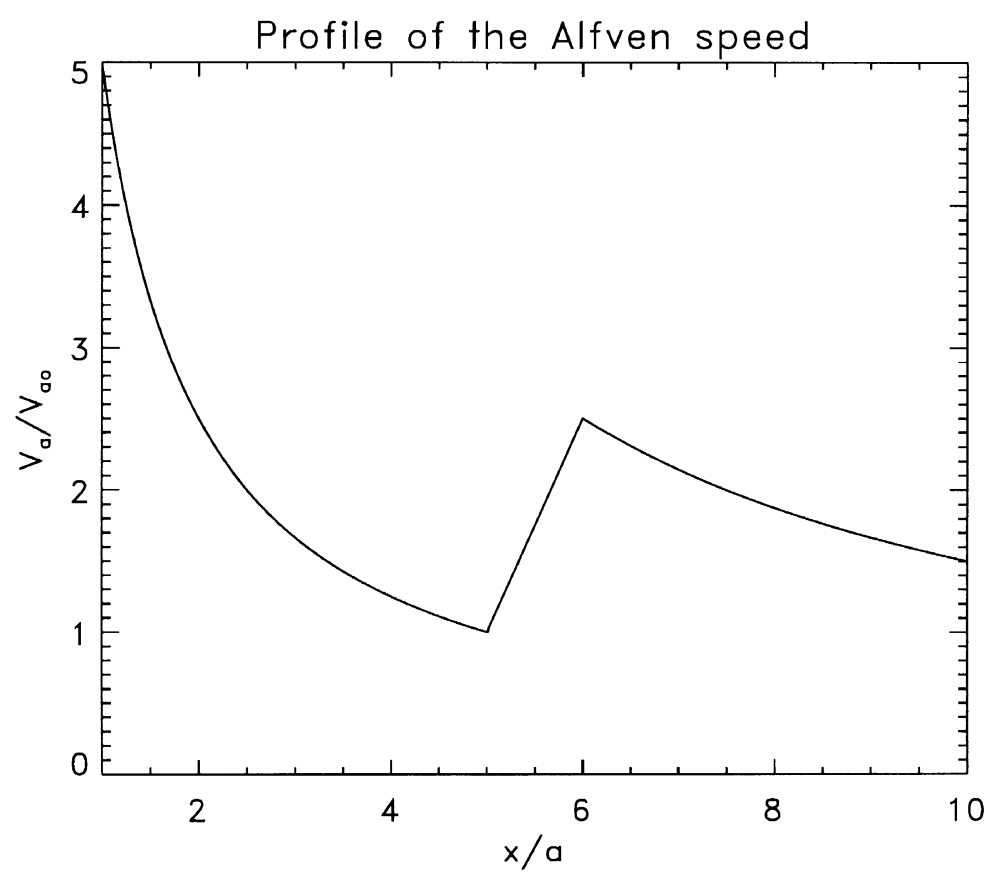

Fig. 2. An example of the profile of the Alfven speed $v_{\mathrm{a}}$ normalized by $v_{\text {ao }}$ at the plasmapause for $p=5, q=6, t=10$ and $R=9$ which is similar to Lin et al. (1991).

for the ionosphere in the south hemisphere. In this study, the ionosphere is assumed as a perfect conductor from which fast compressional waves could reflect perfectly to excite a cavity resonance.

For the case with a cold plasma imbedded in the box model, MHD waves can be derived from the following forms:

$$
\begin{gathered}
\mathbf{E}+\mathbf{V} \times \mathbf{B}=0, \\
\rho \frac{d \mathbf{V}}{d t}=\mathbf{F}-\frac{1}{\mu_{\mathrm{o}}} \mathbf{B} \times(\nabla \times \mathbf{B}), \\
\nabla \times \mathbf{E}=-\frac{d \mathbf{B}}{d t}
\end{gathered}
$$

where $\mathbf{E}$ is the electric field, $\mathbf{B}$ denotes the magnetic field, $\rho$ represents the plasma density, $\mathbf{V}$ is the bulk velocity with zero-order $\mathbf{V}_{\mathrm{o}}=0$ and $\mu_{\mathrm{o}}$ denotes the permeability in the free space. For equilibrium initially, we assume there exists the plasma force $\mathbf{F}$ which equals to the magnetic force $\frac{1}{\mu_{\mathrm{o}}} \mathbf{B}_{\mathrm{o}} \times$ $\left(\nabla \times \mathbf{B}_{\mathrm{o}}\right)$ where $\mathbf{B}_{\mathrm{o}}$ is the ambient magnetic field.

The linearized MHD equation of the perturbed electric field $\mathbf{E}_{1}$ can be obtained from Eqs. (1), (2) and (3):

$$
\begin{aligned}
\frac{d^{2} \mathbf{E}_{1}}{d t^{2}}=\frac{1}{\mu_{\mathrm{o}} \rho_{\mathrm{o}}}\{( & \left(\nabla \times \nabla \times \mathbf{E}_{1}\right) \times \mathbf{B}_{\mathrm{o}} \times \mathbf{B}_{\mathrm{o}} \\
& \left.+\left(\nabla \times \mathbf{B}_{\mathrm{o}}\right) \times\left(\nabla \times \mathbf{E}_{1}\right) \times \mathbf{B}_{\mathrm{o}}\right\}
\end{aligned}
$$

where $\rho_{\mathrm{o}}$ is the ambient plasma density. Since Eq. (1) implies that the perturbed electric field $\mathbf{E}_{1}$ doesn't have $z$ component, $\mathbf{E}_{1}$ can be assumed as

$$
\mathbf{E}_{1}=\left(E_{1 x}(x), E_{1 y}(x), 0\right) \exp [i(m y+k z-\omega t)]
$$

where $E_{1 y}$ is the electric field of the compressional wave and $E_{1 x}$ corresponds to the toroidal component. In addition, $m$ is the azimuthal wave number, $k$ is the wave number along the $z$ axis and $\omega$ is the wave frequency respectively.

Referring to Lin et al. (1991), the ambient plasma density $\rho_{\mathrm{o}}$ is assumed as follows:

$$
\begin{gathered}
\rho_{\mathrm{o}}(x)=\rho_{\mathrm{p}} \cdot p^{4} \cdot\left(\frac{a}{x}\right)^{4} \quad a \leq x \leq p a, \\
\rho_{\mathrm{o}}(x)=\rho_{\mathrm{p}} \cdot p^{6} \cdot\left(\frac{a}{x}\right)^{6} \cdot\left[1+\left(\frac{x}{a}-p\right) \frac{\sqrt{R}-1}{q-p}\right]^{-2} \\
p a<x<q a, \\
\rho_{\mathrm{o}}(x)=\frac{1}{R} \cdot \rho_{\mathrm{p}} \cdot p^{4} \cdot\left(\frac{a}{x}\right)^{4} \\
q a \leq x \leq t a
\end{gathered}
$$

where $\rho_{\mathrm{p}}$ is the plasma density at the plasmapause and $R$ denotes the ratio of the plasma density drop.

In this study, the ambient magnetic field is assumed as straight but varied radially. Thus, the Earth's magnetic field is expressed as follows:

$$
\mathbf{B}_{\mathrm{o}}(x)=B_{\mathrm{p}} \cdot p^{3} \cdot\left(\frac{a}{x}\right)^{3} \hat{z}
$$

where $B_{\mathrm{p}}$ is the magnetic field and $p$ is the $L$ value at the plasmapause.

With Eqs. (6) and (7), the Alfven speed $v_{\mathrm{a}}=B_{\mathrm{o}} / \sqrt{\mu_{\mathrm{o}}} \rho_{\mathrm{o}}$ can be described as follows:

$$
\begin{gathered}
v_{\mathrm{a}}(x)=v_{\mathrm{ao}} \cdot p \cdot \frac{a}{x} \quad a \leq x \leq p a, \\
v_{\mathrm{a}}(x)=v_{\mathrm{ao}} \cdot\left\{1+\left(\frac{x}{a}-p\right) \frac{\sqrt{R}-1}{q-p}\right\} \\
p a<x<q a,
\end{gathered}
$$




$$
m a=0, k a=0.2 \pi, p=5, q=5.1, R=9
$$
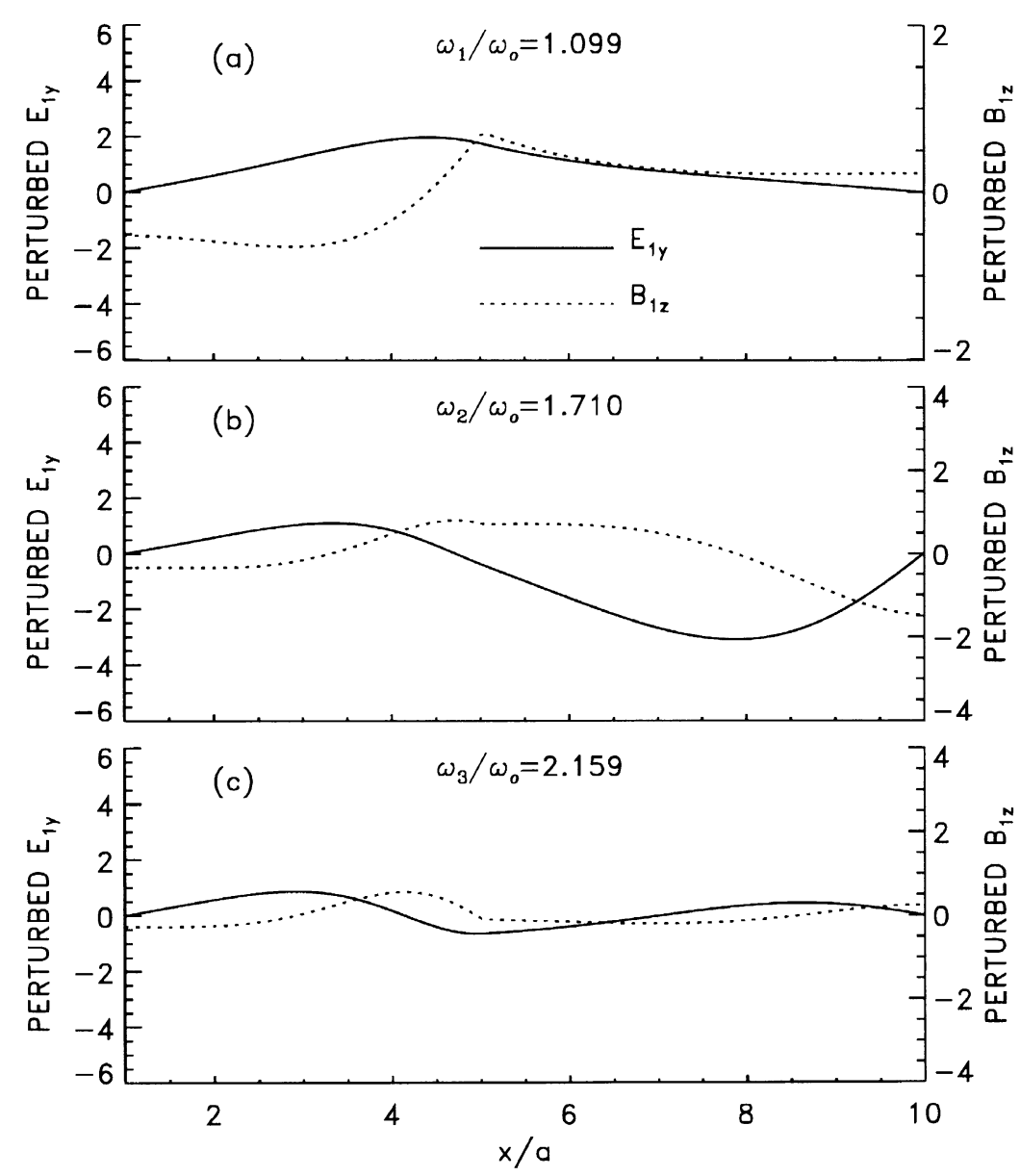

Fig. 3. (a) The profiles of the perturbed electric field $E_{1 y}$ and the perturbed magnetic field $B_{1 z}$ of the fundamental cavity mode in the radial direction obtained from the box magnetosphere for the homogeneous ambient magnetic field with $m a=0, k a=0.2 \pi, p=5, q=5.1, \omega_{1} / \omega_{0}=1.099$ and $R=9 . E_{1 y}$ and $B_{1 z}$ are indicated with the solid line and the dashed line, respectively. (b) Same as Fig. 3(a), except for the second cavity mode and the eigenfrequency $\omega_{2} / \omega_{0}=1.710$. (c) Same as Fig. 3(a), except for the third cavity mode and the eigenfrequency $\omega_{3} / \omega_{0}=2.159$.

$$
v_{\mathrm{a}}(x)=\sqrt{R} \cdot v_{\mathrm{ao}} \cdot p \cdot \frac{a}{x} \quad q a \leq x \leq t a
$$

where $v_{\text {ao }}$ is the Alfven speed at the plasmapause. Values of $p, q, t$ and $R$ are determined by the geomagnetic activity.

Substituting Eqs. (5), (6), (7) and (8) into Eq. (4), we can decompose Eq. (4) into the following equations:

$$
\begin{gathered}
\frac{d^{2} E_{1 y}}{d x^{2}}+\left\{\frac{2 m^{2} \omega^{2}\left(\frac{d v_{\mathrm{a}}}{d x}\right)}{\left(\frac{\omega^{2}}{v_{\mathrm{a}}^{2}}-k^{2}\right)\left(\frac{\omega^{2}}{v_{\mathrm{a}}^{2}}-k^{2}-m^{2}\right) v_{\mathrm{a}}^{3}}\right. \\
\left.+\frac{1}{B_{\mathrm{o}}} \frac{d B_{\mathrm{o}}}{d x}\right\} \frac{d E_{1 y}}{d x} \\
+\left(\frac{\omega^{2}}{v_{\mathrm{a}}^{2}}-k^{2}-m^{2}\right) E_{1 y}=0, \\
E_{1 x}=\frac{-i m \frac{d E_{1 y}}{d x}}{\left(k^{2}+m^{2}-\frac{\omega^{2}}{v_{\mathrm{a}}^{2}}\right)} .
\end{gathered}
$$

Note that Eq. (9) is like the governing equation in the early FLRs theory in which the ambient magnetic field $B_{0}$, was assumed as homogeneous (e.g. Southwood, 1974). Later, it was reintroduced to qualitatively study the wave coupling in the global cavity mode theory (e.g. Kivelson and Southwood, 1986). Moreover, it was used in the recent numerical works to examine the spatial structure of the global cavity mode. By using the finite element method, Krauss-Varban and Patel (1988) were the first to investigate the normal mode in the plasmasphere with consideration of the radially varying magnetic field and the realistic Alfven speed profile. They ignored the term of the varying $B_{\mathrm{o}}$ with an explanation that it does not affect the behavior of the wave coupling. It should be noted that the property of global cavity modes depends on the ambient magnetic field. In order to explain the observation of Pi 2 pulsations at $L=1.06$, Lin et al. (1991) also used the same MHD equations to estimate magnetospheric and plasmaspheric cavity modes respectively. Since $\nabla \times \mathbf{B}_{\mathrm{o}}=0$ in the dipole coordinate, Eq. (4) would become the governing equation in their model which is similar to the classical box model. However, it seems inconsistent that they treated the 


$$
\mathrm{ma}=0, \mathrm{ka}=0.2 \pi, p=5, q=5.1, \mathrm{R}=9
$$
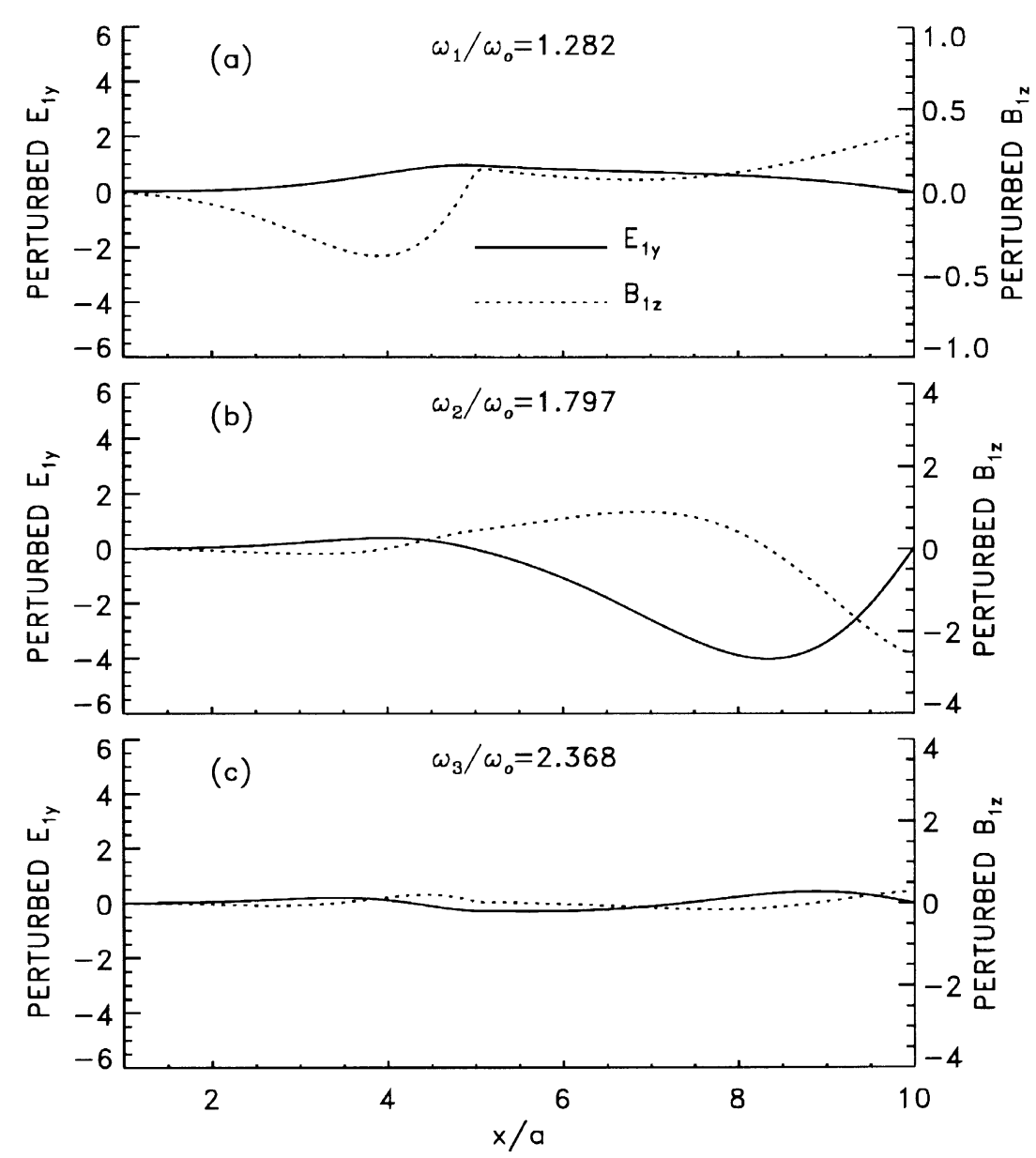

Fig. 4. (a) The profiles of the perturbed electric field $E_{1 y}$ and the perturbed magnetic field $B_{1 z}$ of the fundamental cavity mode in the radial direction obtained from the box magnetosphere for the inhomogeneous ambient magnetic field with $m a=0, k a=0.2 \pi, p=5, q=5.1, \omega_{1} / \omega_{0}=1.282$ and $R=9$. $E_{1 y}$ and $B_{1 z}$ are indicated with the solid line and the dashed line, respectively. (b) Same as Fig. 4(a), except for the second cavity mode and the eigenfrequency $\omega_{2} / \omega_{0}=1.797$. (c) Same as Fig. 4(a), except for the third cavity mode and the eigenfrequency $\omega_{3} / \omega_{0}=2.368$.

ambient magnetic field as a transformation of the dipole field for the equatorial plane and kept it straight like the classical box model. On the other hand, it should be noted that Eq. (9) will be singular as $\omega^{2}=v_{\mathrm{a}}^{2} k^{2}$ and $\omega^{2}=v_{\mathrm{a}}^{2}\left(k^{2}+m^{2}\right)$ for the azimuthal wave number $m \neq 0$. For $\omega^{2}=v_{\mathrm{a}}^{2} k^{2}$, there is a singularity corresponding to a point where FLR would occur as the nature resonance frequency of field lines matches the frequency of fast compressional waves. For $\omega^{2}=v_{\mathrm{a}}^{2}$ $\left(k^{2}+m^{2}\right)$, there is a singularity corresponding to the turning point where fast compressional waves decay with distance in the inner magnetosphere. As $m \neq 0$, Eq. (10) can be used to calculate the toroidal component $E_{1 x}$ and shows the existence of toroidal oscillations. Physically, it means that the wave coupling would occur as the wave frequency meets. Despite recent observations interpreted $\mathrm{Pi} 2$ pulsations as associated with a global cavity resonance, early ground measurements showed that azimuthal wave numbers of Pi 2 pulsations have a latitudinal dependence (see review by Yumoto, 1986; and references there in). This means that the azimuthal wave number $m$ may be not exactly zero. Owing to the singularity in the governing equation, cavity modes estimated by Lin et al. (1991) are appropriate for $m=0$. In other words, the coupling of cavity modes to FLRs may be a possible source mechanism for Pi 2 pulsations in addition to cavity resonances. To clarify the source mechanism of Pi 2 pulsations, it is necessary to reexamine Eq. (4) by taking into account both the inhomogeneity of the ambient magnetic field and the singularity problem for $m \neq 0$. In order to quantitatively compare with the observational result, the theoretical model will be studied with the realistic Alfven speed profile in the homogeneous and inhomogeneous ambient magnetic field, respectively.

\section{Numerical Results}

For a complete mathematical treatment of singularity, we should use Laplace transformation and derive a Green's function for the coupled equations (e.g. Zhu and Kivelson, 1988). But as Fujita and Patel (1992) noted, it is quite difficult to formulate the Green's function in the realistic magnetosphere. Hence, like Fujita and Patel (1992), we perform normal modes analysis to study the coupled oscillations in the box model. Basically, there are two physical means to 

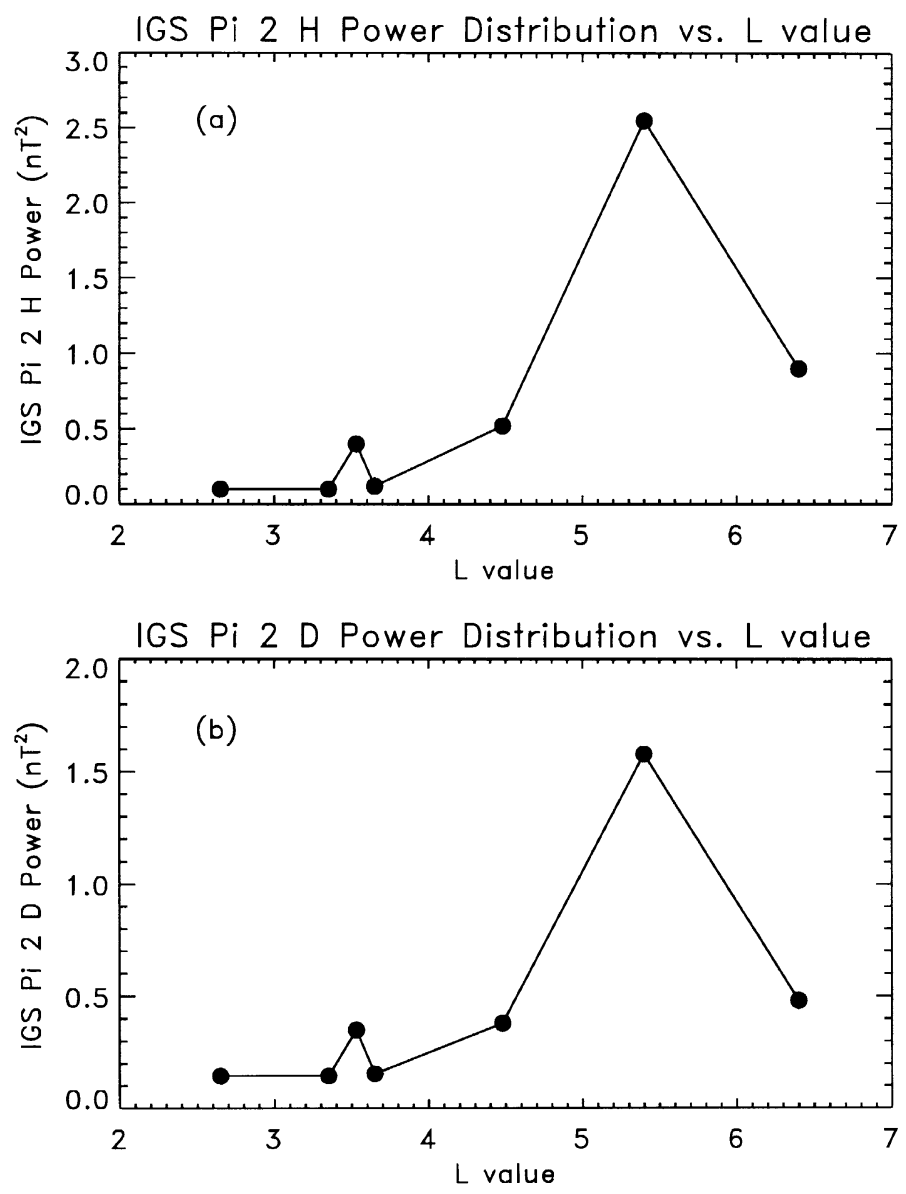

Fig. 5. (a) The power of the filtered $H$ component of Pi 2 versus $L$ value in IGS chain during March-April in 1978 and 1979 . (b) Same as Fig. 5(a), except for the power of the filtered $D$ component of Pi 2 .

remove the singularity in Eq. (9) for $m \neq 0$ : the irreversible wave coupling of cavity modes to FLRs by adopting a complex frequency and the ionospheric dissipation by adopting a complex wave number. In general, the energy dissipation of a fast magnetospheric cavity mode is a combination of at least three possible mechanisms: the ionospheric Joule dissipation, the Poynting flux, and the wave coupling. It is hard to determine quantitatively which mechanism is the most important one responsible for the energy dissipation. By using radar measurements and ground magnetometers, Crowley et al. (1987) found that the damping rate of cavitymode pulsations is controlled by the damping of a cavity mode rather than the ionospheric Joule dissipation from the FLR. This implies that the ionospheric Joule dissipation might be insignificant in damping the cavity mode. On the other hand, there is no rigid boundary except for the Earth in the real magnetosphere to reflect fast compressional waves back to the magnetospheric cavity. Even for an apparent boundary like the dayside magnetopause, the perfect reflection is not easy for fast compressional waves propagating from the magnetosphere to the magnetosheath (Fujita and Patel, 1992). Therefore, some part of the wave energy in the magnetosphere will be transmitted through the magnetospheric boundary. This means that the Poynting flux flows across the magnetospheric boundary might represent the en- ergy loss of a fast magnetospheric cavity mode. Since the ionospheric Joule dissipation occurs mainly in a small area around the footpoint of resonant field line but the Poynting flux flows across almost the entire magnetospheric boundary, Fujita and Patel (1992) qualitatively suggested that the Poynting flux might be more significant in damping coupled global oscillations. If the Poynting flux is responsible for the energy dissipation of a fast magnetospheric cavity mode, the wave number should be a complex. Because fast compressional waves interact with the ionosphere in a different way to shear Alfven waves, it is impossible to choose a single value of the imaginary component of the wave number which is appropriate for both near the resonance and away from the resonance (Mann et al., 1995). In order to avoid unrealistic effects, the coupling of a fast magnetospheric cavity mode to FLRs could be investigated by adopting a complex frequency rather than a complex wave number. In this study, the wave coupling is assumed as irreversible for the damping of a fast magnetospheric cavity mode.

In the classical FLR theory, the frequency of hydromagnetic perturbation is assumed as a complex with a negative imaginary $\omega_{\mathrm{i}}$. Similarly, $\gamma=\omega_{\mathrm{i}} / \omega_{\mathrm{r}}$ denotes the damping of fast magnetospheric cavity modes and $\omega_{\mathrm{r}}$ for the real part of the complex wave frequency. Referring to Zhu and Kivelson (1988), the normal modes of a system can be obtained with 


$$
\mathrm{ma}=0.01, \mathrm{ka}=0.2 \pi, \mathrm{p}=5.7, \mathrm{q}=6.2, \mathrm{R}=9, \omega_{\mathrm{r}} / \omega_{0}=1.036, \gamma=-0.00152
$$

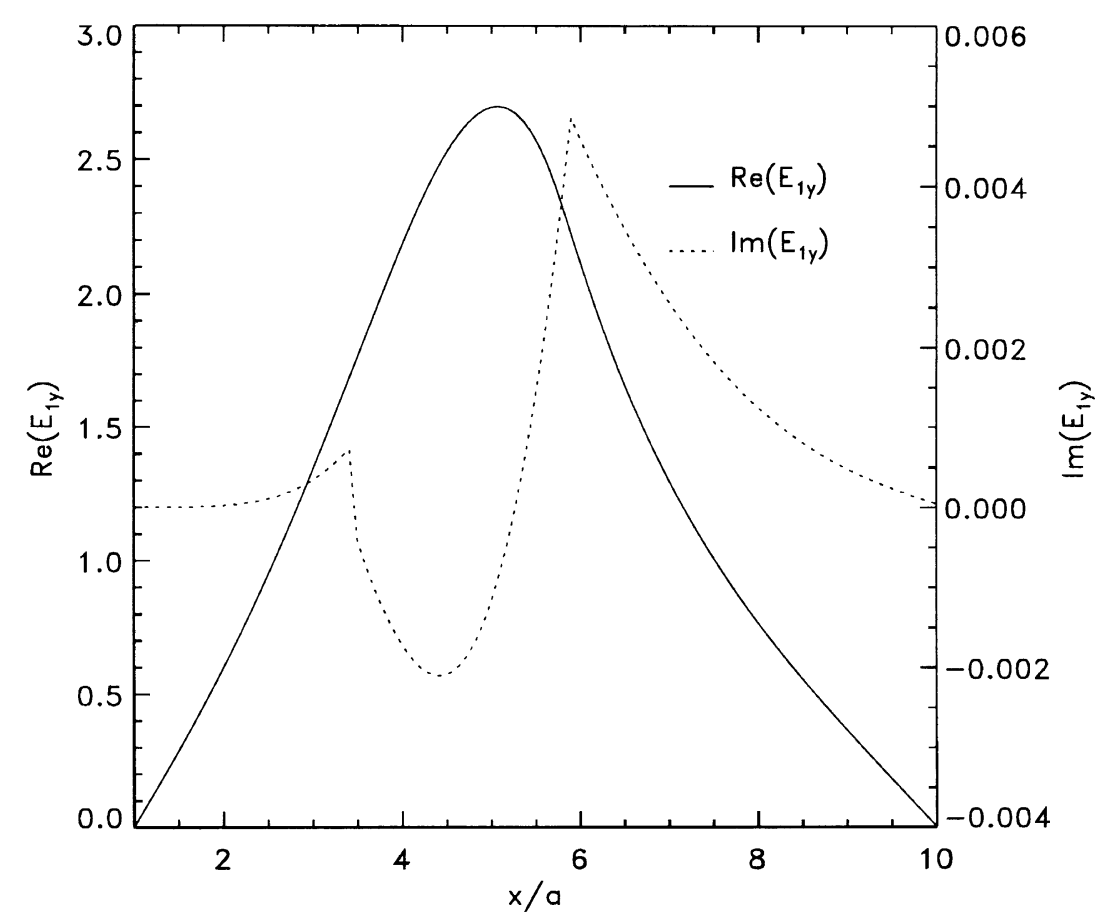

Fig. 6. The profile of the perturbed electric field $E_{1 y}$ for the homogeneous ambient magnetic field with $m a=0.01, k a=0.2 \pi, p=5.7, q=6.2, R=9$, $\omega_{\mathrm{r}} / \omega_{\mathrm{o}}=1.036$ and $\gamma=-0.00152$. The solid curve is for the real part of $E_{1 y}$ and the dashed curve for the imaginary part of $E_{1 y}$.

two standard approaches. One approach is that the normal modes are inferred from the Green's function. The other approach is that the time variable is Fourier transformed and the properties of the normal modes are obtained as the eigenfunction and eigenvalues of a differential equation. We addressed that it is hard to derive the Green's function in the realistic magnetosphere. Hence, the coupled global oscillations is studied with the latter approach. In this study, the electric perturbation $E_{1 y}$ in Eq. (9) is solved as a complex function with discrete $\omega_{\mathrm{r}}$ and $\omega_{\mathrm{i}}$ which are determined by the boundary condition. At the substorm expansive onset, the density of plasma injection seems highly populated like the ionosphere. Hence, $x=t a$ corresponds to the position of plasma injection fronts which could be regarded as a perfect conductor at the substorm expansive onset. For fast compressional waves propagating between the low latitude ionosphere and plasma injection fronts, $E_{1 y}=0$ at $x=a$ and $x=t a$ are assumed as the boundary condition (cf. Kivelson and Southwood, 1988). In other words, same as $\omega_{\mathrm{r}}$ and $\omega_{\mathrm{i}}$, the damping factor $\gamma=\omega_{\mathrm{i}} / \omega_{\mathrm{r}}$ is discrete for satisfying the boundary condition that both the real part and the imaginary part of $E_{1 y}$ are simultaneously zero at $x=a$ and $x=t a$. Following Lin et al. (1991), we solve Eq. (9) by adopting the fourth order Runge-Kutta method to estimate magnetospheric cavity modes. Then, $E_{1 x}$ can be calculated by taking $E_{1 y}$ into Eq. (10). The magnetic perturbation can be acquired by substituting $E_{1 y}$ and $E_{1 x}$ into Eq. (3).

Before performing the calculation of the eigenfrequency and eigenfunction of magnetospheric cavity modes for $m \neq$ 0 , we first study the theoretical model for $m=0$. In this study, both the Alfven speed $v_{\mathrm{a}}$ and the phase speed $v=\omega / k$ of hydromagnetic perturbations are normalized by $v_{\mathrm{ao}}$. For convenience of calculation, the wave length and the grid size are normalized by the Earth's radius $a$. The wave number $k$ can be expressed as $k a=n \pi / l, n=1,2,3, \ldots$ where $l=5$ is the normalized length of the magnetic field line (see Fig. 1). Henceforth, $m a$ denotes the azimuthal wave number $m$. The Alfven speed profile shown in Fig. 2 is similar to the profile given by Lin et al. (1991) for $p=5, q=6, t=10$ and $R=9$. In this study, cavity modes are calculated for $n=1$ with reference to in-phase of $B_{1 x}$ component of Pi 2 pulsations on AMPTE/CCE in both hemispheres (Takahashi et al., 1995). It is shown in Fig. 3 that $E_{1 y}$ and $B_{1 z}$ of three cavity modes in the homogeneous ambient magnetic field which are the same as Lin et al. (1991) for $k a=0.2 \pi, m a=$ $0, p=5, q=5.1, t=10$ and $R=9$. Note that $\omega_{\mathrm{o}}=v_{\mathrm{ao}} / a$, $\omega_{n}$ is the frequency of magnetospheric cavity modes and the subscript $n=1,2,3$ denotes the first three harmonic number. Figure 4 shows that $E_{1 y}$ and $B_{1 z}$ of three cavity modes in the inhomogeneous ambient magnetic field are calculated with the same parameters in Fig. 3. By comparing Figs. 3 and 4 , the frequency of cavity modes apparently depends on the ambient magnetic field. In addition, the magnetic perturbation $B_{1 z}$ becomes zero rather than finite at the inner boundary in Fig. 4.

The identification of Pi 2 events generally relies on $H$ and $D$ components of magnetic perturbations. Hence, the power of Pi 2 pulsations is dominated by $H$ and $D$ components. In order to clarify the substorm mechanism, Hsu and McPherron (1996) constructed the Pi 2 power in IGS chain by using the 
$m a=0.01, k a=0.2 \pi, p=5.7, q=6.2, R=9, \omega_{r} / \omega_{0}=1.036, \gamma=-0.00152$
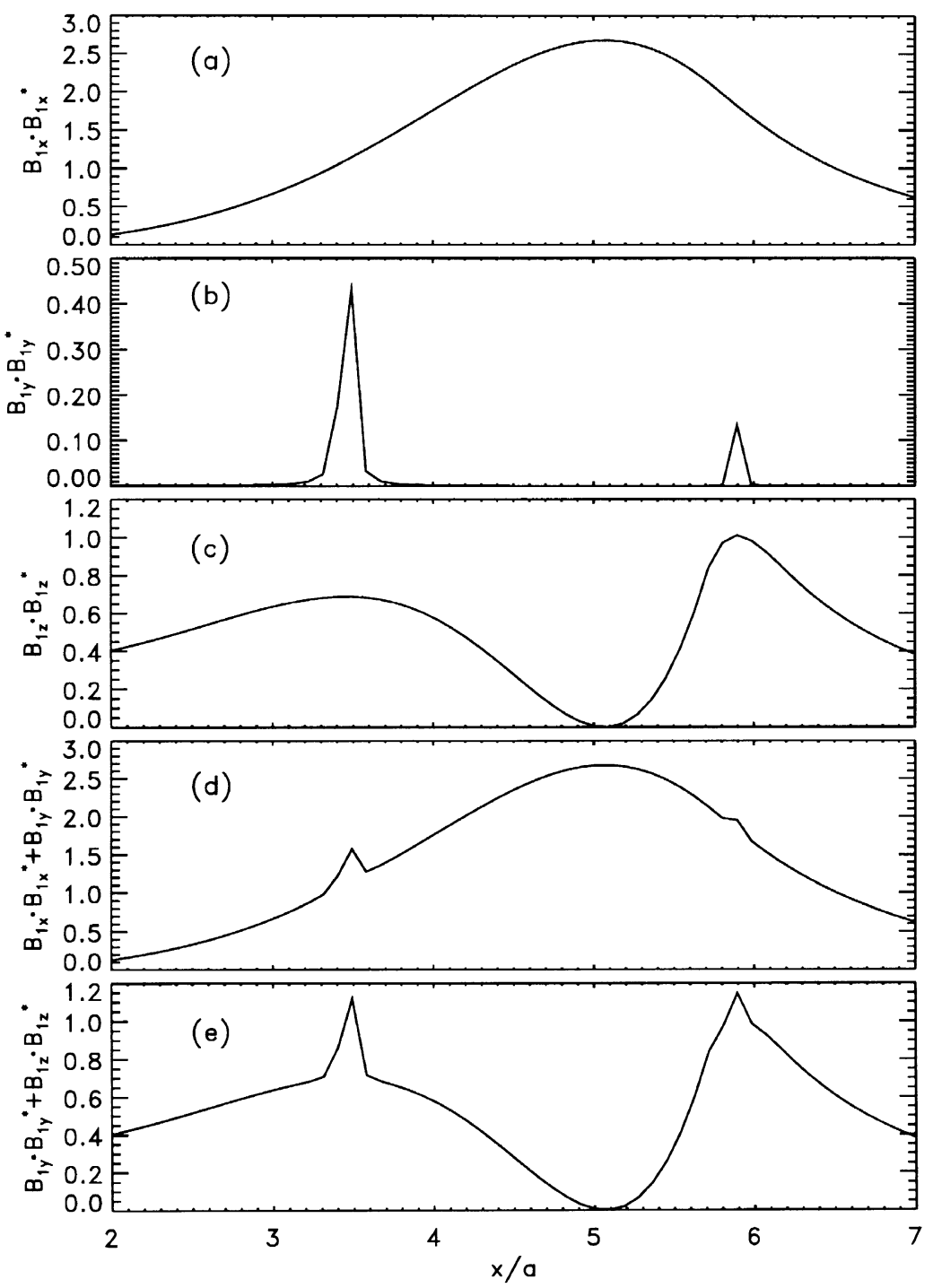

Fig. 7. (a) Same as Fig. 6, except for $B_{1 x} \cdot B_{1 x}^{*}$. (b) Same as Fig. 6, except for $B_{1 y} \cdot B_{1 y}^{*}$. (c) Same as Fig. 6, except for $B_{1 z} \cdot B_{1 z}^{*}$. (d) Same as Fig. 6, except for the addition of both $B_{1 x} \cdot B_{1 x}^{*}$ and $B_{1 y} \cdot B_{1 y}^{*}$. (e) Same as Fig. 6, except for the addition of both $B_{1 y} \cdot B_{1 y}^{*}$ and $B_{1 z} \cdot B_{1 z}^{*}$.

combination of the power of filtered $H$ and $D$ components. They utilized the Pi 2 power to identify the onset time for a substorm event in their database. Referring to their observational results, the power of the filtered $H$ component in IGS is shown in Fig. 5(a) and the power of the filtered $D$ component is in Fig. 5(b), respectively. It is noteworthy in Figs. 5(a) and 5(b) that two enhancements are located at $L=3.5$ and 5.5, respectively. Moreover, it is shown in Figs. 5(a) and 5(b) that the filtered $D$ component is comparable to the filtered $H$ component for the power enhancement around $L=3.5$. This result implies that the FLR might be responsible for the power enhancement around $L=3.5$. For the ambient magnetic field vertical to the ionosphere, Hughes and Southwood (1976) proposed that magnetic signals on the ground is rotated through $90^{\circ}$ with respect to the magnetic field of the transverse mode (toroidal mode) in the magnetosphere. There is also a view point that $H$ and $D$ components of geomagnetic pulsations may be the mixture of the com- pressional component and the transverse component of MHD waves in the magnetosphere under the different ionospheric condition (e.g. Allan et al., 1996). In this study, $B_{1 x}$ and $B_{1 z}$ are the compressional components of MHD waves and $B_{1 y}$ is the transverse component. With no availability of in situ measurements of the ionosphere above IGS chain, the ionosphere is assumed as a thin film. As a result, the power of $H$ component at the resonance site is assumed to be the addition of $B_{1 x} \cdot B_{1 x}^{*}$ and $B_{1 y} \cdot B_{1 y}^{*}$. The magnetic perturbation with superscript “*” represents it's complex conjugate. As for $D$ component, it's power is assumed to be the addition of $B_{1 y} \cdot B_{1 y}^{*}$ and $B_{1 z} \cdot B_{1 z}^{*}$. But away from the resonance site, the power of $H$ and $D$ components are assumed to be the combination of some part of $B_{1 x} \cdot B_{1 x}^{*}, B_{1 y} \cdot B_{1 y}^{*}$ and $B_{1 z} \cdot B_{1 z}^{*}$. In fact, the ionosphere above IGS chain is permeated with the obliquely incident ambient magnetic fields. In addition, the ionosphere above the neutral atmosphere is really a thick layer with conductivity and not a thin film at all. Therefore, 


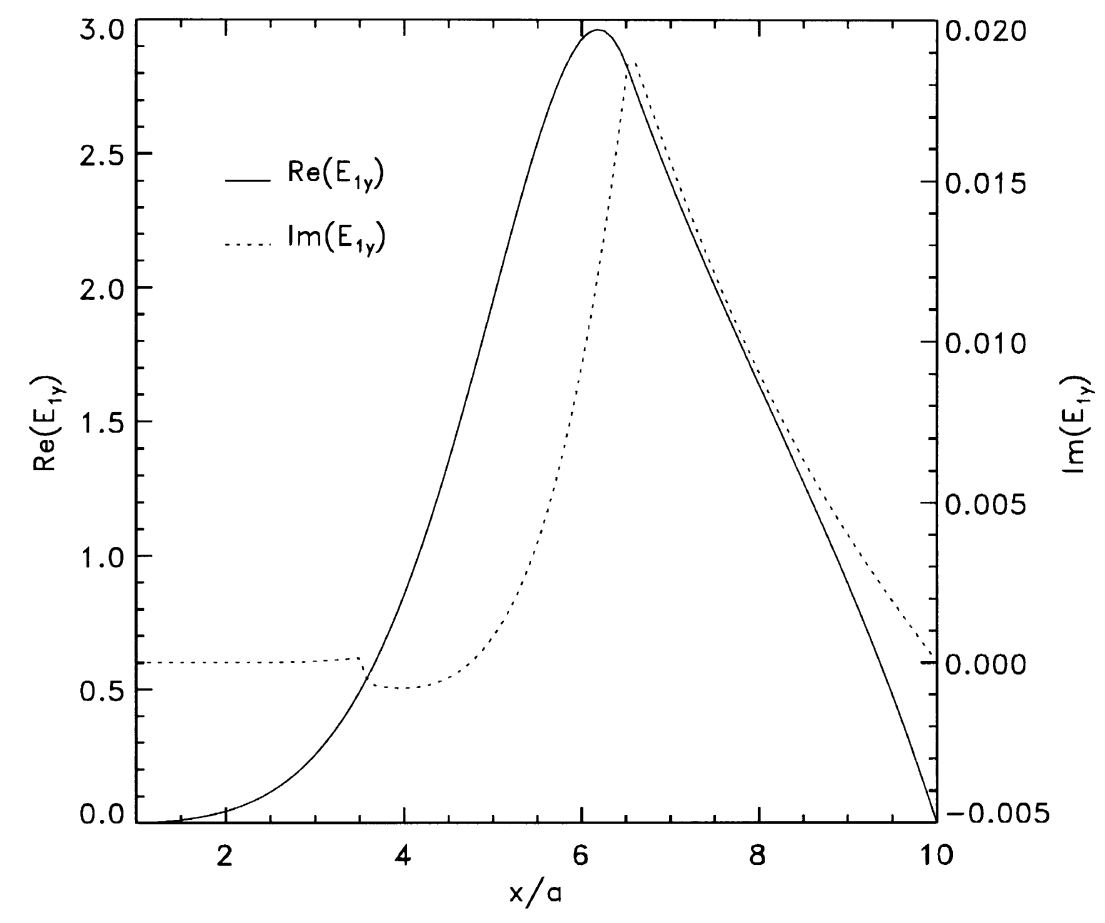

Fig. 8. The profile of the perturbed electric field $E_{1 y}$ for the inhomogeneous ambient magnetic field with $m a=0.01, k a=0.2 \pi, p=6.5, q=6.6$, $R=9, \omega_{\mathrm{r}} / \omega_{\mathrm{o}}=1.144$ and $\gamma=-0.0022$. The solid curve is for the real part of $E_{1 y}$ and the dashed curve for the imaginary part of $E_{1 y}$.

by taking into account the ionospheric conductivity, it is another interesting topic for the future study to investigate the ionospheric property through mapping the magnetic perturbation from the magnetosphere to the ground.

To find out which source mechanism is responsible for the aforementioned phenomenon, we attempt to calculate the fundamental cavity mode in the homogeneous ambient magnetic field for appropriate parameters. Note that $x / a$ in this study corresponds to $L$ value. The electric perturbation $E_{1 y}$ of a fundamental cavity mode is calculated for $k a=$ $0.2 \pi, m a=0.01, p=5.7, q=6.2, \omega_{\mathrm{r}} / \omega_{\mathrm{o}}=1.036$, $\gamma=-0.00152, t=10$ and $R=9$. In Fig. 6, the solid curve is for the real part of $E_{1 y}$ and the dashed curve for the imaginary part of $E_{1 y}$. For a fundamental cavity mode, it is evident in Fig. 6 that both the real part and the imaginary part of $E_{1 y}$ become zero at $x / a=1$ and $x / a=10$. The power distributions of $B_{1 x} \cdot B_{1 x}^{*}, B_{1 y} \cdot B_{1 y}^{*}$ and $B_{1 z} \cdot B_{1 z}^{*}$ are plotted in the Figs. 7(a), 7(b) and 7(c) for same parameters in Fig. 6. It is noteworthy that there are two enhancements in Fig. 7(b) near $x / a=3.5$ and 5.9, respectively. Figure 7(d) shows that the addition of both $B_{1 x} \cdot B_{1 x}^{*}$ and $B_{1 y} \cdot B_{1 y}^{*}$ has two enhancements at $x / a=3.5$ and 5.0. With combination of $B_{1 y} \cdot B_{1 y}^{*}$ and $B_{1 z} \cdot B_{1 z}^{*}$, there are two enhancements at $L=3.5$ and 5.9 in Fig. 7(e). For the inhomogeneous ambient magnetic field, the fundamental cavity mode is recalculated for $k a=0.2 \pi, m a=0.01, p=6.5, q=6.6, \omega_{\mathrm{r}} / \omega_{\mathrm{o}}=$ 1.144, $\gamma=-0.0022, t=10$ and $R=9$. Same as Fig. 6, Fig. 8 illustrates the profile of both the real part and the imaginary part of $E_{1 y}$. It is shown in Fig. 9(b) that there is an enhancement at $x / a=3.6$ in the $B_{1 y} \cdot B_{1 y}^{*}$ profile. However,
Fig. 9(d) displays two enhancements at $x / a=3.6$ and 6.1 with combination of $B_{1 x} \cdot B_{1 x}^{*}$ and $B_{1 y} \cdot B_{1 y}^{*}$. It is shown in Fig. 9(e) that there are two enhancements at $x / a=3.6$ and 5.1 with combination of $B_{1 y} \cdot B_{1 y}^{*}$ and $B_{1 z} \cdot B_{1 z}^{*}$. In contrast, the profile of $B_{1 x} \cdot B_{1 x}^{*}$ and $B_{1 y} \cdot B_{1 y}^{*}$ or $B_{1 y} \cdot B_{1 y}^{*}$ and $B_{1 z} \cdot B_{1 z}^{*}$ in Figs. 7 and 9 are quite similar to the power distribution of filtered $H$ or $D$ components of Pi 2 pulsations in IGS chain. Moreover, the damping factor $\gamma$ in Figs. 7 and 9 is consistent with the damping rate of Pi 2 pulsations analyzed with the maximum likelihood method by Itonoga et al. (1992).

\section{Evidence of the Coupling of a Magnetospheric Cavity Mode to FLRs}

To justify whether both Figs. 7 and 9 could explain the power distribution of filtered $H$ and $D$ components of $\mathrm{Pi}$ 2 pulsations in IGS chain, we have to estimate the period of the fundamental cavity mode with specific parameters. Lin et al. (1991) derived a formula to estimate the period of magnetospheric cavity modes

$$
T=\frac{59 \times 10^{-6} p^{3}\left(m_{\mathrm{i}} / m_{\mathrm{p}}\right)^{1 / 2}\left(n_{\mathrm{o}}\right)^{1 / 2}}{\omega_{\mathrm{c}}} \mathrm{s}
$$

where $m_{\mathrm{i}} / m_{\mathrm{p}}$ is the ratio of the average ion mass to the proton mass, $n_{\mathrm{o}}$ is the plasma number density at the plasmapause and $\omega_{\mathrm{c}}=\omega_{\mathrm{r}} / \omega_{\mathrm{o}}$ is the normalized frequency of magnetospheric cavity modes. For the homogeneous ambient field, the corresponding period of the fundamental cavity mode obtained by taking $\omega_{\mathrm{c}}=1.036, p=5.7, m_{\mathrm{i}} / m_{\mathrm{p}}=7.2$ and $n_{\mathrm{o}}=9 \times 10^{6} \mathrm{~m}^{-3}$ into Eq. (11) is about $T=87 \mathrm{~s}$. Similarly, the corresponding period $T=116 \mathrm{~s}$ is obtained from Eq. 


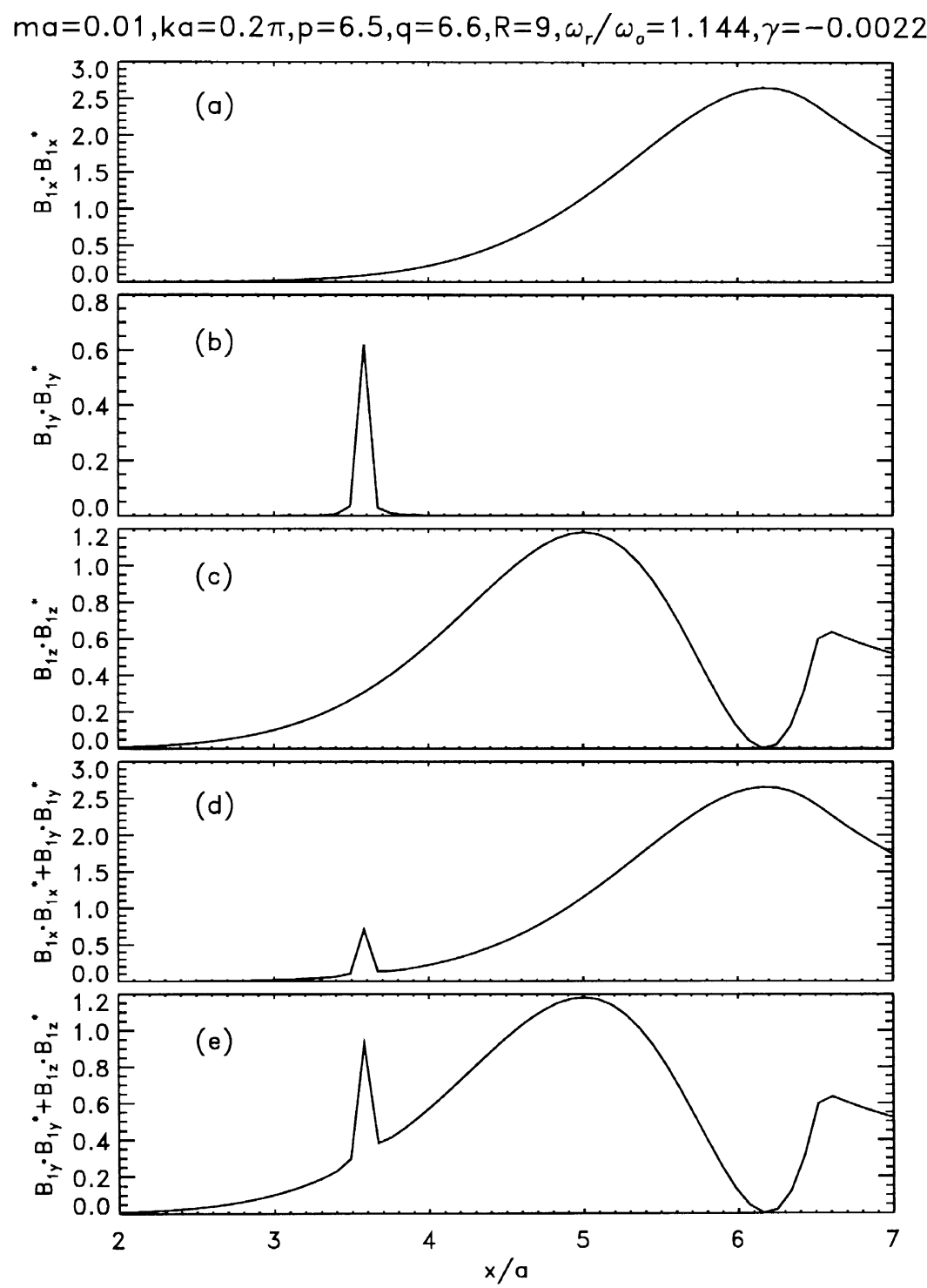

Fig. 9. (a) Same as Fig. 8, except for $B_{1 x} \cdot B_{1 x}^{*}$. (b) Same as Fig. 8, except for $B_{1 y} \cdot B_{1 y}^{*}$. (c) Same as Fig. 8, except for $B_{1 z} \cdot B_{1 z}^{*}$. (d) Same as Fig. 8, except for the addition of both $B_{1 x} \cdot B_{1 x}^{*}$ and $B_{1 y} \cdot B_{1 y}^{*}$. (e) Same as Fig. 8, except for the addition of both $B_{1 y} \cdot B_{1 y}^{*}$ and $B_{1 z} \cdot B_{1 z}^{*}$.

(11) by taking $\omega_{\mathrm{c}}=1.144$ and $p=6.5$ with the same value of $m_{\mathrm{i}} / m_{\mathrm{p}}$ and $n_{\mathrm{o}}$ for the inhomogeneous ambient field. It is obvious that the period of the fundamental cavity mode in Figs. 7 and 9 is in the period range of Pi 2 pulsations.

Next, we examine whether the enhancements in the power distribution of filtered $H$ and $D$ components of Pi 2 pulsations in IGS chain result from the coupling of a magnetospheric cavity mode to FLRs. In this study, the eigenfrequency of the oscillating magnetic field line can be estimated with Alfven travel time (e.g. Orr, 1973) and expressed as

$$
\omega_{\mathrm{b}}=\frac{\pi \cdot v_{\mathrm{a}} \cdot \omega_{\mathrm{o}}}{l} .
$$

Figure 7(b) has two enhancements near $x / a=3.5$ and 5.9 where the Alfven speed $v_{\mathrm{a}}$ can be acquired from the Alfven speed profile similar to Fig. 2 for $p=5.7, q=6.2, R=9$. By taking the Alfven speed $v_{\mathrm{a}}$ of interest into Eq. (12), the eigenfrequency of the magnetic field line can be estimated and matches the eigenfrequency of the fundamental cavity mode in Fig. 7. Hence, two power enhancements at $x / a=3.5$ and 5.9 in Figs. 7(d) and 7(e) result from FLR. But the power enhancement at $x / a=5.0$ results from the fundamental cavity mode. With substitution of the Alfven speed $v_{\mathrm{a}}$ in Fig. 9 into Eq. (12), it can be found out that a power enhancement at $x / a=3.6$ results from the FLR but other power enhancements at $x / a=6.1$ in Fig. 9(d) and at $x / a=5.1$ in Fig. 9(e) are the cavity mode instead. The resonance sites in Figs. 7 and 9 are not exactly the same as the power distribution of Pi 2 pulsations in IGS chain. Early ground radar detection (Walker et al., 1979) and recent satellite observation (Mitchell et al., 1990) showed that the resonance width is about $0.5 a$. Since shear Alfven waves can carry the field aligned current to the ionosphere but not for fast compressional waves, the ionospheric conductivity at a small area around the foot point of resonant field line may be enhanced as the FLR occurs. Hence, the resonance width $0.5 a$ may correspond to a small area around the foot point 
Pi 2 Burst Events ( $H$ component) on 04/05/78
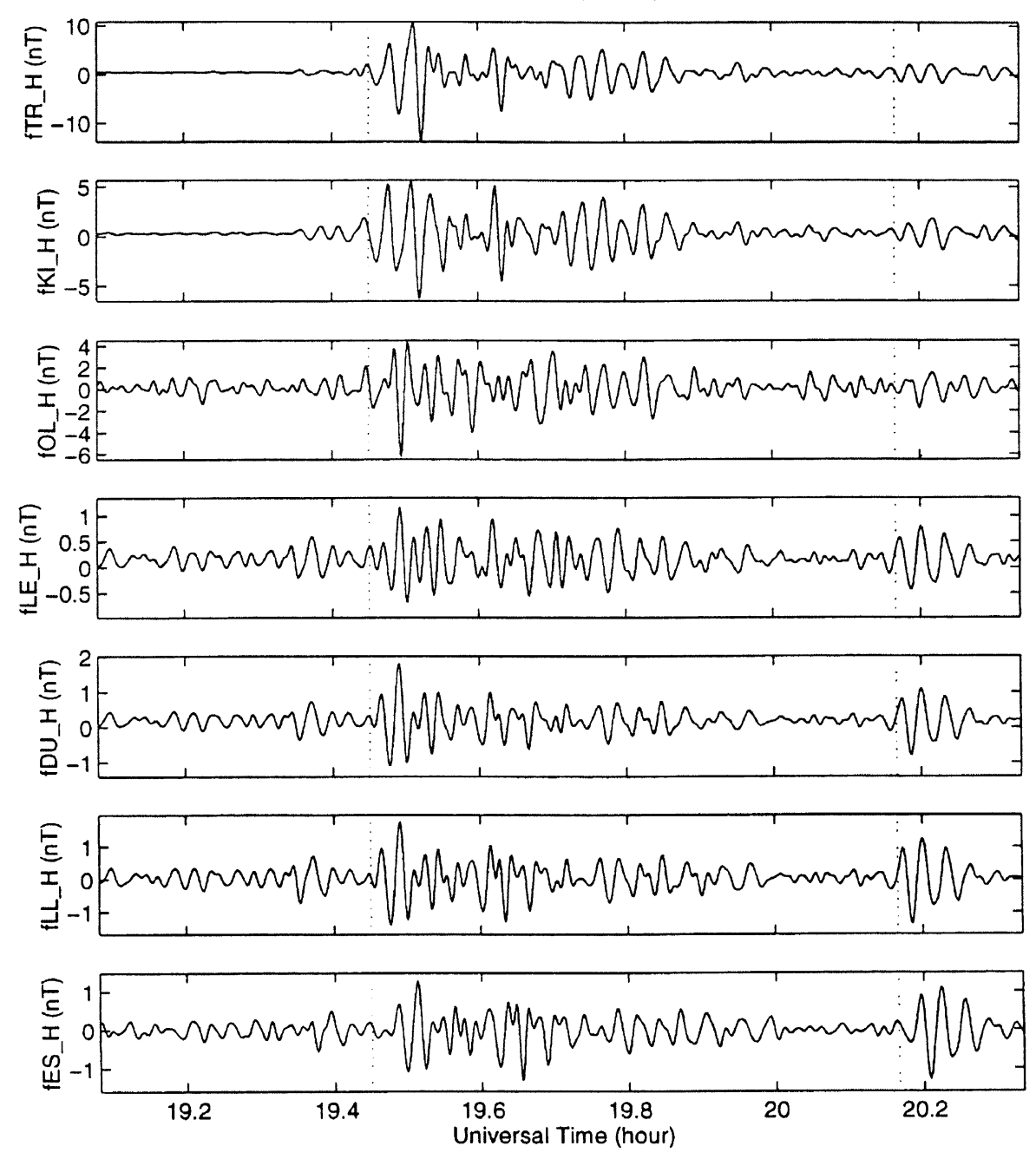

Fig. 10. An example of filtered $H$ components of Pi 2 in IGS chain on April 5, 1978. There are two Pi 2 burst events which occur at 19:30 UT and 20:10 UT, marked with the dotted line, respectively.

of resonant field line excited by coupled global oscillations. On the other hand, ULF waves appear in wave packet and with banded frequency. It might result in an edge around resonant field line at which the nature resonance frequency no longer matches the wave frequency. With allowance of the resonance width, the power enhancements of filtered $H$ and $D$ components in IGS chain may result from FLRs.

In order to clarify the power enhancements of Pi 2 pulsations around $L=3.5$ and 5.5 which may be caused by the ionospheric and/or crustal conductivity anomaly, we choose a typical example of Pi 2 pulsations on April 5, 1978 to analyze the phase structure. In Fig. 10, seven panels illustrate the filtered $H$ components in IGS chain from high $L$ to low $L$. Figure 11 shows that the filtered $D$ components in IGS chain are plotted in sequence from the top to the bottom as Fig. 10. There are two Pi 2 burst events in Figs. 10 and 11 which occur at 19:30 UT and 20:10 UT (marked with the dotted line), respectively. By referring to Beamish et al. (1979), the phase of filtered $H$ and $D$ components are analyzed with a complex demodulation technique in which the phase is calculated for the dominant spectral peak based on the Fast Fourier Transform. The latitudinal phase structure is plotted in Fig. 12(a) (for 19:30 UT event) and Fig. 12(b) (for 20:10 UT event), respectively. As shown in Figs. 12(a) and $12(\mathrm{~b})$, the triangle denotes $H$ component and the cross denotes $D$ component. It is shown in Figs. 12(a) and 12(b) that the out-of-phase (corresponding to the phase shift $180^{\circ}$ ) occur apparently around $L=3.5$ for both events. But around $L=5.5$, the phase change seems not obvious for both events. According to the global cavity mode theory, the out-of-phase would occur around the resonant position. Hence, there is a possibility that the power enhancements of filtered $H$ and $D$ components around $L=3.5$ result from the coupling of a cavity mode to FLR.

In Figs. 7 and 9, the power enhancement around $L=3.5$ may result from the FLR, and the fundamental cavity mode, which is in Pi 2's band, is represented by a broad power peak which occupies an extended latitude from $L=2$ to $L=7$. Accordingly, we suggest that the power enhancements of filtered $H$ and $D$ components of Pi 2 pulsations in IGS chain may be an evidence of the coupling of a fast magnetospheric cavity mode to FLRs. 
Pi 2 Burst Events (D component) on 04/05/78
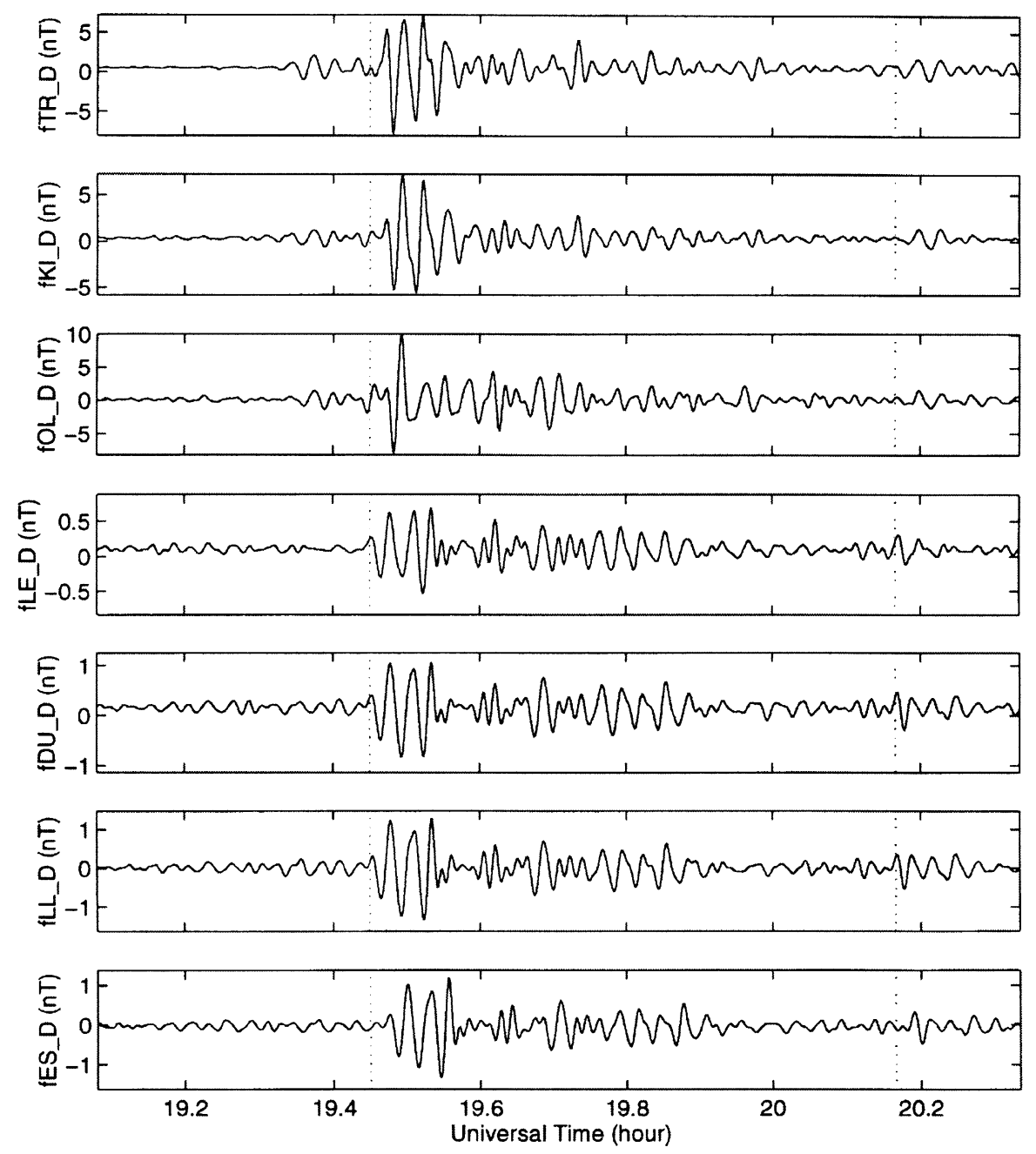

Fig. 11. An example of filtered $D$ components of Pi 2 in IGS chain on April 5, 1978. Same as $H$ component in Fig. 8, two Pi 2 burst events apparently occur at 19:30 UT and 20:10 UT, respectively.

\section{Discussion and Summary}

There is a trend in Figs. 7 and 9 that the magnitude of $B_{1 y} \cdot B_{1 y}^{*}$ is smaller than $B_{1 x} \cdot B_{1 x}^{*}$ and $B_{1 z} \cdot B_{1 z}^{*}$ except for the resonance sites. This trend is consistent with observational results at AMPTE/CCE analyzed by Takahashi et al. (1995). After driven by plasma injection fronts at the substorm expansive onset, the cavity mode becomes dominant in the inner magnetosphere and resonant oscillations of local field line would be excited due to the matching of eigenfrequency. This scenario is similar to one proposed by Yumoto et al. (1989). In this study, the irreversible coupling of a fast magnetospheric cavity mode to FLRs is assumed if the Poynting flux flows across the magnetospheric boundary is the main process of the energy dissipation. It is noteworthy that the magnitude of $B_{1 y} \cdot B_{1 y}^{*}$ is comparable to the compressional components $B_{1 x} \cdot B_{1 x}^{*}$ and $B_{1 z} \cdot B_{1 z}^{*}$ as the FLR occurs at low $x / a$. With the addition of $B_{1 x} \cdot B_{1 x}^{*}$ and $B_{1 y} \cdot B_{1 y}^{*}$ for $H$ component as well as by adding $B_{1 y} \cdot B_{1 y}^{*}$ and $B_{1 z} \cdot B_{1 z}^{*}$ for $D$ component, the first power enhancement around $L=3.5$ in Figs. 7 and 9 can be attributed to the coupling of a fast magnetospheric cavity mode to FLRs. For high $x / a$ value in Fig. 7, the magnitude of the compressional component $B_{1 x} \cdot B_{1 x}^{*}$ and $B_{1 z} \cdot B_{1 z}^{*}$ is larger than the toroidal component $B_{1 y} \cdot B_{1 y}^{*}$. Hence, it is shown in Fig. 7 that the second power enhancement is mainly dominated by the compressional mode. But the second power enhancement in Fig. 9 apparently results from the compressional mode with no existence of the toroidal mode. By comparing Figs. 7 and 9, the coupling of a fast magnetospheric cavity mode to the FLR can be identified unambiguously for low $x / a$ value. Though the resonance site and the frequency of cavity modes depend on the ambient magnetic field, it is shown in Figs. 7 and 9 that the ambient magnetic field would not change the behavior of the coupling of a fast magnetospheric cavity mode to FLRs. However, it is found in Fig. 4 that the magnetic perturbation $B_{1 z}$ becomes zero rather than finite at the inner boundary. Such a numerical result is inconsistent with observations by Takahashi et al. (1992). They found that $\mathrm{Pi} 2$ pulsations have almost identical amplitudes at Kakioka $(L=1.2)$ and in the inner magnetosphere $(L \sim 3)$. For lack of the curvature and ionospheric effects, the box model with the radial inhomogeneity of the ambient magnetic field $B_{0}$ should be carefully 

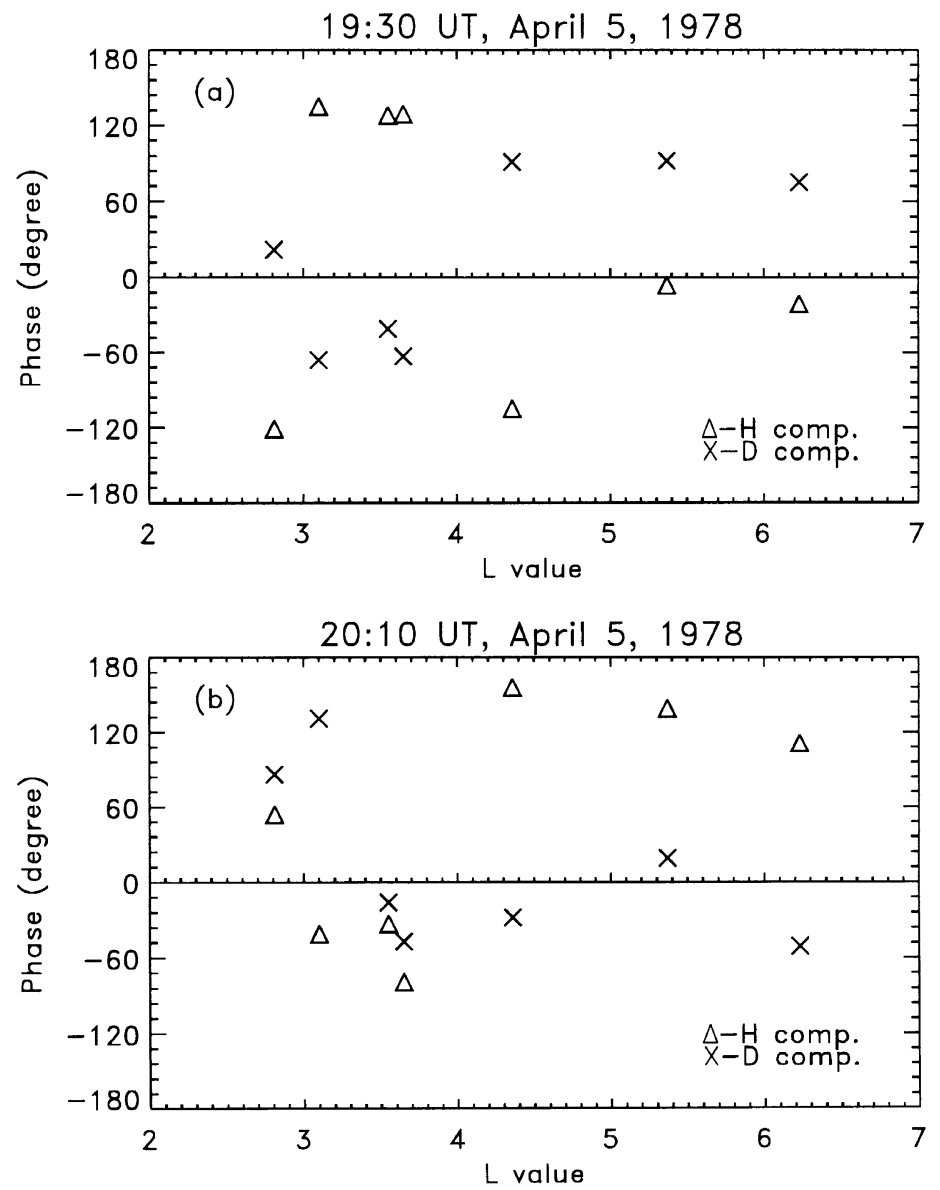

Fig. 12. (a) A latitudinal phase structure of filtered $H$ and $D$ components of Pi 2 at 19:30 UT on April 5, 1978. The triangle denotes $H$ component; The cross denotes $D$ component. The out-of-phase changes apparently occur around $L=3.5$ for both $H$ and $D$ components. (b) Same as Fig. 10(a) except for at 20:10 UT.

used to explain the observations at $L \leq 3$. Most of IGS chain are located beyond $L=3$ except for one at $L=2.7$. Thus, the box model could provide the physical insight of coupled global oscillations at $L>3$ with consideration of the radial inhomogeneity of the ambient magnetic field.

The second power enhancement of Pi 2 pulsations in IGS chain is around $L=5.5$ where is approximately the location of the plasmapause. Sutcliff (1975) proposed that the source mechanism of mid-latitude Pi 2 pulsations might be associated with the surface waves at the plasmapause. Hence, the second power enhancement may be attributed to the surface waves at the plasmapause. To verify whether the second power enhancement results from the surface waves, we apply a formula $T_{\mathrm{s}}=2 l / \sqrt{2} v_{\mathrm{a}}$ proposed by Hasegawa and Chen (1974) to estimate the period of surface waves. In Figs. 7 and 9 , the periods of surface waves are about $T_{\mathrm{s}}=89 \mathrm{~s}$ and $81 \mathrm{~s}$ which do not match the period of a fundamental cavity mode. It is apparent that the second power enhancement around $L=5.5$ in IGS chain may not be associated with the surface waves at the plasmapause.

The power enhancements of Pi 2 pulsations in IGS chain may be a local phenomenon due to enhanced conductivity in the ionosphere. For auroral regions, the ionospheric conductivity in the nighttime may become enhanced after the substorm onset. The polarization of mid and low latitudes Pi 2 pulsations are closely related to the substorm current wedge (Lester et al., 1983). Lanzerotti and Medford (1984) found out that Pi 2's at low latitudes can't be explained with the FLRs theory and they suggested the possible source mechanism could be the substorm electrojet current wedge. Since some stations in IGS chain are located near the aurora region, the second power enhancement may be attributed to the locally enhanced conductivity brought by the substorm current wedge. The substorm current wedge consists of the field aligned current which is driven by Alfven waves traveling along the auroral zone field lines. By comparing the spacecraft AMPTE/CCE and ground observations, statistical analysis of $\mathrm{Pi} 2$ pulsations in the inner magnetosphere by Takahashi et al. (1995) show no evidence to support a view that ground Pi 2 pulsations at mid-latitudes $(2<L<5)$ are resonant oscillations in response to the source waves on the auroral zone field line. While the orbit of AMPTE/CCE was in the nightside, the amplitude of the azimuthal component corresponding to the toroidal component is smaller than the compressional component. As a consequence, their results implied that mid-latitudes Pi 2 pulsations observed on the ground originates from the cavity resonance excited in the inner magnetosphere. However, the latitudinal phase 
structure of Pi 2 pulsations in IGS chain shows that there is a probable resonant site around $L=3.5$ where the phase change seems out-of-phase. For lack of observations from spaced multi-spacecraft, the toroidal component comparable to the compressional component may not be easily found out. Hence, it can't rule out that the coupling of a fast magnetospheric cavity mode to FLRs is a possible source mechanism for low-latitudes Pi 2 pulsations. The well-coordinated simultaneous measurements of $\mathrm{Pi} 2$ pulsations on the ground and in space are needed for further verification of the source mechanism. On the other hand, since no crustal conductivity in IGS chain is available, it also can't rule out that the crustal conductivity anomaly may result in the power enhancements of Pi 2 pulsations in IGS chain. Hence, more ground observations are also needed to verify whether the crustal conductivity anomaly plays an important role on the power enhancements of Pi 2 pulsations in IGS chain.

This study is to present an evidence of the coupling of a fast magnetospheric cavity mode to FLR. The power of filtered $H$ and $D$ components of $\mathrm{Pi} 2$ pulsations during March-April in 1978 and 1979 from IGS chain in UK shows that there are two enhancements located near $L=3.5$ and 5.5 in the distribution profile. The irreversible hydromagnetic wave coupling is studied by using a box model for the magnetosphere. The cold linearized MHD equations is examined with realistic Alfven speed profile for the uniform and nonuniform ambient magnetic fields, respectively. With appropriate parameters to depict the magnetospheric environments during the aforementioned period, numerical results are acquired with the fourth order Runge-Kutta method and quite consistent with data analysis. We suggest that the coupling of a fast magnetospheric cavity mode driven by fast compressional waves owing to the impulsive source at the magnetotail to resonant field line at low latitudes is a possible scenario of the propagation mechanism for low latitude Pi 2 pulsations.

Acknowledgments. The first author thanks C. A. Lin, W. H. Tsai and L. H. Lyu at National Central University in Taiwan for useful discussions and encouragement. The authors would like to thank L. Chen from University of California at Irvine for constructive suggestions. This work was supported by National Science Council of R.O.C. in Taiwan under grant NSC 87-2111-M-008-AP8.

\section{References}

Allan, W. and E. M. Poulter, ULF waves-their relationship to the structure of the Earth's magnetosphere, Rep. Prog. Phys., 55, 533-598, 1992.

Allan, W., S. P. White, and E. M. Poulter, Impulsive-excited hydromagnetic cavity and field-line resonances in the magnetosphere, Planet. Space Sci., 34, 371-385, 1986.

Allan, W., F. W. Menk, B. J. Fraser, Y. Li, and S. P. White, Are low-latitude Pi 2 pulsations cavity/waveguide modes?, Geophys. Res. Lett., 23, 765-768, 1996.

Beamish, D., H. W. Hanson, and D. C. Webb, Complex demodulation applied to Pi 2 geomagnetic pulsations, Geophys. J. R. astr. Soc., 58, 471493, 1979

Chen, L. and A. Hasegawa, A theory of long-period magnetic pulsations, 1, steady state excitation of field line resonances, J. Geophys. Res., 79, 1024-1032, 1974.

Crowley, G., W. J. Hughes, and T. B. Jones, Observational evidence of cavity modes in the Earth's magnetosphere, J. Geophys. Res., 92, 12233-12240, 1987.

Fairfield, D. H., Advances in magnetospheric storm and substorm research: 1989-1991, J. Geophys. Res., 97, 10865-10874, 1992.

Feng, Q., B. J. Fraser, F. W. Menk, C. W. S. Ziesolleck, O. Saka, and T. Kitamura, Pc 3-4 geomagnetic pulsations observed at very low latitude conjugate stations, J. Geophys. Res., 100, 19287-19298, 1995.

Fujita, S. and V. L. Patel, Eigenmode analysis of coupled magnetohydrodynamic oscillations in the magnetosphere, J. Geophys. Res., 97, 1377713788, 1992.

Hasegawa, A. and L. Chen, Theory of magnetic pulsations, Space Sci. Rev., 16, 347-359, 1974.

Hsu, T. S. and R. L. McPherron, Occurrency frequency of substorm field and plasma signatures observed near-Earth by ISEE-1/2, in Proceeding of the 3rd International Conference on Substorms, Versailles, Paris, May 1996.

Hughes, W. J. and D. J. Southwood, The screening of micropulsation signals by the atmosphere and ionosphere, J. Geophys. Res., 81, 3234-3240, 1976.

Itonaga, M., T.-I. Kitamura, O. Saka, H. Tachihara, M. Shinohara, and A. Yoshikawa, Discrete spectral structure of low-latitude and equatorial $\mathrm{Pi}$ 2 pulsation, J. Geomag. Geoelectr., 44, 253-259, 1992.

Jacobs, J. A., Y. Kato, S. Matsushita, and V. A. Troitskaya, Classification of geomagnetic micropulsations, J. Geophys. Res., 69, 180-181, 1964.

Kivelson, M. G. and D. J. Southwood, Coupling of Global magnetospheric MHD eigenmodes to field line resonances, J. Geophys. Res., 91, 43454351, 1986.

Kivelson, M. G. and D. J. Southwood, Hydromagnetic waves and the ionosphere, Geophys. Res. Lett., 15, 1271-1274, 1988.

Krauss-Varban, D. and V. L. Patel, Numerical analysis of the coupled hydromagnetic waves equations in the magnetosphere, J. Geophys. Res., 93, 9721-9729, 1988.

Lanzerotti, L. J. and L. V. Medford, Local night, impulsive (Pi 2-type) hydromagnetic wave polarization at low latitudes, Planet. Space Sci., 32, 135-142, 1984.

Lee, D. H., Dynamics of MHD waves propagation in the low-latitude magnetosphere, J. Geophys. Res., 101, 15371-15386, 1996.

Lester, M., W. J. Hughes, and H. J. Singer, Polarization patterns of Pi 2 magnetic pulsations and the substorm current wedge, J. Geophys. Res., 88, 7958-7966, 1983.

Lin, C. A., L. C. Lee, and Y. J. Sun, Observations of Pi 2 pulsations at a very low latitude ( $L=1.06)$ station and magnetospheric cavity resonances, $J$. Geophys. Res., 96, 21105-21114, 1991.

Mann, I. R., A. N. Wright, and P. S. Cally, Coupling of magnetospheric cavity modes to field line resonances: a study of resonances width, $J$ Geophys. Res., 100, 19441-19456, 1995.

McPherron, R. L., C. T. Russel, and M. P. Aubry, Satellite studies of magnetospheric substorms on August 15, 1968, Phenomenological model for substorm, J. Geophys. Res., 78, 3131-3149, 1973.

Mitchell, D. G., M. J. Engbreston, D. J. Williams, C. A. Cattell, and R. Lundin, Pc 5 pulsations in the outer dawn magnetosphere seen by ISEE 1 and 2, J. Geophys. Res., 95, 967-975, 1990.

Orr, D., Magnetic pulsations within the magnetosphere: A review, J. Atmos. Terr. Phys., 35, 1-50, 1973.

Saito, T., K. Yumoto, and Y. Koyama, Magnetic pulsation Pi 2 as a sensitive indicator of magnetospheric substorm, Planet. Space Sci., 24, 1025-1029, 1976.

Samson, J. C. and G. Rostoker, Latitude-dependent characteristics of high latitude Pc 4 and Pc 5 micropulsations, J. Geophys. Res., 77, 6133-6144, 1972.

Southwood, D. J., Some features of field line resonance in the magnetosphere, Planet. Space Sci., 22, 483-491, 1974.

Stuart, W. F., Array of Magnetometers in NW Europe, The IMS Source Book, edited by C. T. Russel and David J. Southwood, 141pp., 1982.

Sutcliffe, P. R., The association of harmonics in Pi 2 power spectra with the plasmapause, Planet. Space Sci., 23, 1581-1587, 1975.

Takahashi, K., S. I. Ohtani, and K. Yumoto, AMPTE CCE observations of Pi 2 pulsations in the inner magnetosphere, Geophys. Res. Lett., 19 , 1447-1450, 1992.

Takahashi, K., S. I. Ohtani, and B. J. Anderson, Statistical analysis of Pi 2 pulsations observed by the AMPTE CCE spacecraft in the inner magnetosphere, J. Geophys. Res., 100, 21,929-21,941, 1995.

Walker, A. D. M., R. A. Greenwald, W. F. Stewart, and C. A. Green, Stare auroral radar observations of Pc 5 geomagnetic pulsations, J. Geophys. Res., 84, 3373-3388, 1979.

Yeoman, T. K. and D. Orr, Phase and spectral power of mid-latitude Pi 2 pulsations: evidence for a plasmasphere cavity resonances, Planet. Space Sci., 37, 1367-1383, 1989.

Yeoman, T. K., M. P. Freeman, G. D. Reeves, M. Lester, and D. Orr, A comparison of mid-latitude $\mathrm{Pi} 2$ pulsations and geostationary orbit particle injections as substorm indicators, J. Geophys. Res., 99, 4085-4093, 1994. 
Yumoto, K., Generation and propagation mechanisms of low-latitude magnetic pulsations-A review, J. Geophys. Res., 60, 79-105, 1986.

Yumoto, K., K. Takahashi, T. Saito, F. W. Menk, B. J. Fraser, T. A. Potemra, and L. J. Zanetti, Some aspects of the relation between Pi 1-2 magnetic pulsations observed at $L=1.3-2.1$ on the ground and substormassociated magnetic field variations in the near-Earth magnetotail observed by AMPTE/CCE, J. Geophys. Res., 94, 3611-3618, 1989.

Zhu, X. M. and M. G. Kivelson, Analytic formulation and quantitative solutions of the coupled ULF wave problem, J. Geophys. Res., 93, 8602-
$8612,1988$.

Zhu, X. M. and M. G. Kivelson, Global mode ULF pulsations in a magnetosphere with a nonmonotonic Alfven velocity profile, J. Geophys. Res., 94, 1479-1485, 1989

C.-C. Cheng (e-mail: cccheng@sparc.nhit.edu.tw), J.-K. Chao (e-mail: jkchao@jupiter.ss.ncu.edu.tw), and T.-S. Hsu (e-mail: thsu@galsun.igpp. ucla.edu) 